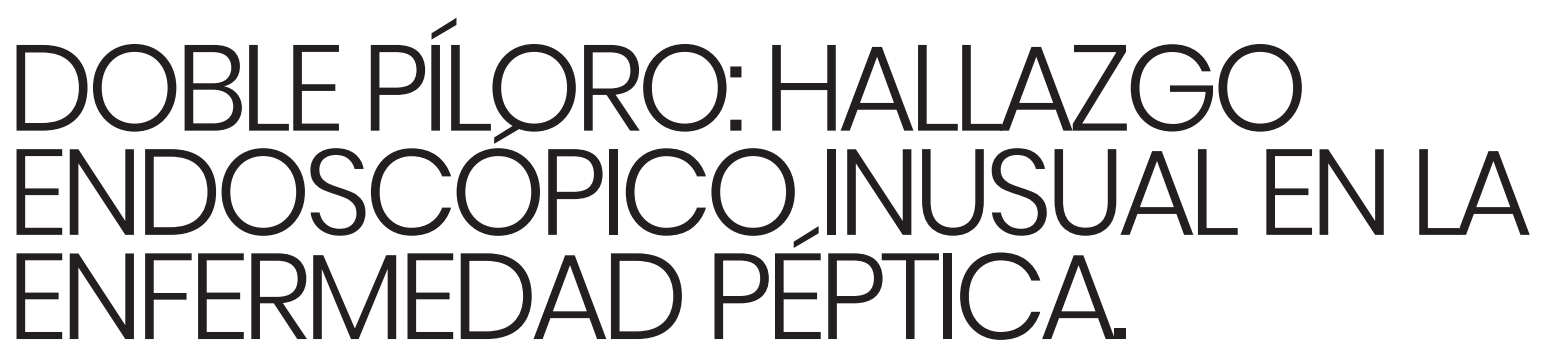

\title{
DOUBLE PYLORUS: UNUSUAL ENDOSCOPIC FINDING IN PEPTIC DISEASE.
}

\section{Díaz Alcázar MM, Ruiz Rodríguez AJ, Martín-Lagos Maldonado A}

Hospital Universitario San Cecilio. Granada.

\section{Resumen}

Hombre de 59 años que consulta por melenas y vómitos en posos de café. En tratamiento con heparina desde una semana antes. Se realiza endoscopia digestiva alta objetivando doble píloro (Figura 1) y úlcera Forrest lla de $2 \mathrm{~cm}$ en cara anterosuperior de bulbo (Figura 2).

El doble píloro o fístula gastroduodenal es un hallazgo endoscópico raro. Se debe a la formación de una fístula entre el estómago y el duodeno. Su incidencia es menor del $0.4 \%$. Como en el caso presentado, habitualmente se forma en el lado de la curvatura menor. Puede ser congénito o adquirido. El doble píloro congénito es extremadamente raro. El doble píloro adquirido se relaciona con la enfermedad péptica gástrica. En la mayoría de los casos la fístula no cierra aunque cicatrice la úlcera.

Palabras clave: doble píloro, úlcera, enfermedad péptica.

\section{Abstract}

A 59-year-old male consults for melena and vomiting similar to coffee grounds. He has been in treatment with heparin for a week. Upper endoscopy is performed, showing double pylorus (Figure 1) and an ulcer Forrest lla, of $2 \mathrm{~cm}$, on the anterosuperior wall of the duodenum (Figure 2).

The double pylorus or gastroduodenal fistula is a rare endoscopic finding. It is due to the formation of a fistula between the stomach and duodenum. Its incidence is less than $0,4 \%$. As in our case, it usually appears on the side of the minor curvature. It can be congenital or acquired. The congenital double pylorus is extremely rare. The acquired double pylorus is associated with the gastric peptic disease. In most cases, the fistula remains open even if the ulcer disappears.

Keywors: double pylorus, ulcer, peptic disease.

\section{Cuerpo}

El doble píloro es un hallazgo endoscópico raro. Se debe a

María del Mar Díaz Alcázar

Hospital Universitario San Cecilio

18016 Granada

mmardiazalcazar@gmail.com

Fecha de envío:02/02/2020

Fecha de aceptación: 15/02/2020 ación de una fístula entre el estómago y el duodeno ${ }^{1-3}$.

Hombre de 59 años sin antecedentes de interés que consulta por melenas y vómitos en posos de café, sin otra sintomatología. Estable hemodinámicamente. En tratamiento con heparina a 
dosis terapéuticas desde una semana antes por trombosis venosa profunda. Se realiza endoscopia digestiva alta objetivando doble píloro (Figura 1) y úlcera Forrest lla de $2 \mathrm{~cm}$, en cara anterosuperior de bulbo (Figura 2). Se trata con inyección de agente esclerosante y colocación de hemoclips.

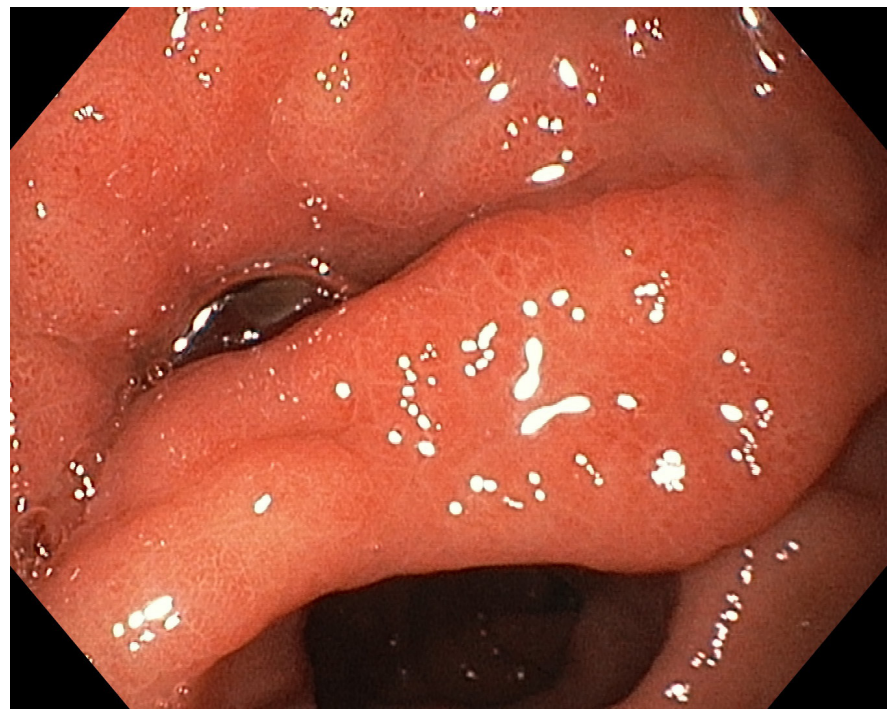

Figura 1

Imagen de endoscopia digestiva alta en que se observa antro con mucosa eritematosa y edematosa y doble píloro.

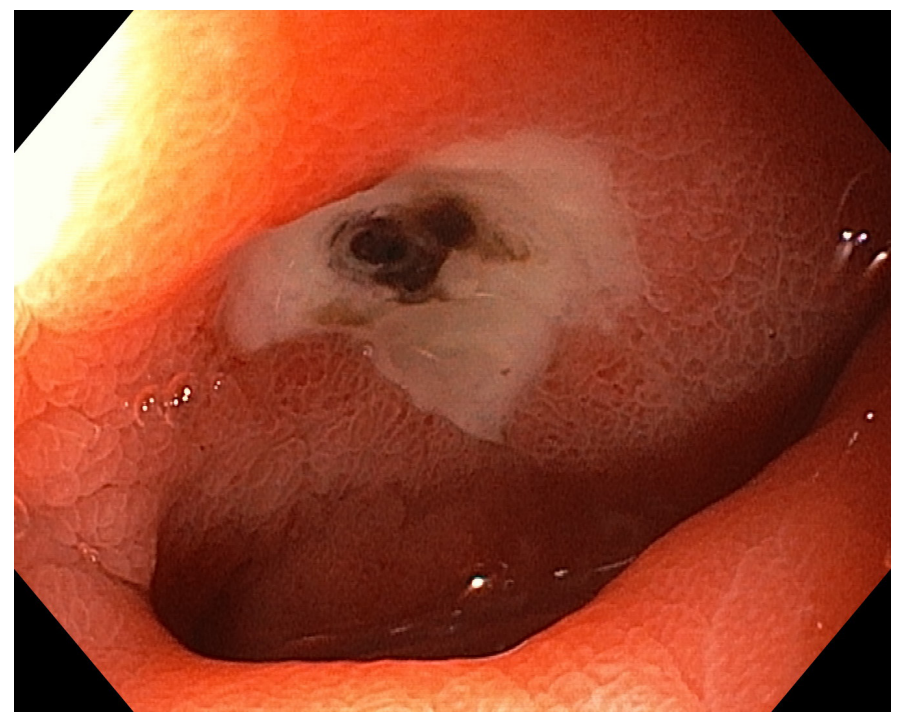

Figura 2

Imagen de endoscopia digestiva alta en que se objetiva la úlcera duodenal desde la fístula gastroduodenal o doble píloro.
El doble píloro o fístula gastroduodenal tiene una incidencia menor del $0,4 \%{ }^{3}$. Es dos veces más frecuente en hombres ${ }^{3}$. Habitualmente se forma en el lado de la curvatura menor, como en el caso presentado ${ }^{1,3}$. La fístula suele formarse con el bulbo, aunque también se ha descrito con la cuarta porción duodenal². El tamaño de la fístula puede variar de milímetros a centímetros, y tiene capa mucosa y muscular ${ }^{2}$. Se debe hacer diagnóstico diferencial con un divertículo gástrico ${ }^{3}$.

El doble píloro puede ser congénito o adquirido ${ }^{1}$. El doble píloro congénito es extremadamente raro, y se debe a un error de canalización durante el desarrollo embrionario precoz ${ }^{2,3}$. Se ha relacionado con la duplicación gástrica o duodenal ${ }^{1,3,4}$. Es un hallazgo incidental que no requiere tratamiento ${ }^{3}$.

El doble píloro adquirido se relaciona con la enfermedad péptica gástrica, y más raramente con úlceras duodenales o cáncer gástrico ${ }^{1-3}$. Según una de las teorías se debe a dos úlceras separadas, una en la curvatura menor del estómago y otra en duodeno, que convergen ${ }^{2,4}$. Habitualmente aparece en mayores de 50 años con ingesta prolongada de AINES o corticoides, o comorbilidades como diabetes, enfermedad renal crónica o enfermedad pulmonar obstructiva crónica, cirrosis hepática, fallo cardíaco o lupus, que afectan a la microcirculación y dificultan la cicatrización ${ }^{1,3}$. Los pacientes suelen tener sintomatología como dolor o sangrado gastrointestinal $\left.\right|^{2,3}$. Se debe investigar la infección por Helicobacter Pylori, y tratar en caso de positividad ${ }^{3}$. En la mayoría de los casos la fístula no cierra aunque cicatrice la úlcera ${ }^{1,3}$.

En algunos casos puede complicarse con obstrucción gástrica ${ }^{3}$. Se podría tratar endoscópicamente con un esfinterotomo, cortando el puente tisular ${ }^{3}$. La cirugía se reserva para el fallo de tratamiento endoscópico, reflujo biliar o perforación ${ }^{3}$.

\section{Bibliografía}

1. Erturk M. Double pylorus; a complication of gastric ulcer. Rev Esp Enferm Dig 2012;104(6):338-339.

2. Durgakeri P, Sarkar A. Double pylorus from a chronic gastric ulcer: an interesting and rare case. ANZ J Surg 2015;85(11):884-886.

3. Fousekis F, Aggeli P, Kotsaftis $P$, et al. Double pylorus: report of a case with endoscopic follow-up and review of the literature. Gastroenterology Res 2018;11(2):154-156.

4. López Casillas N, López-Gaxiola R. Píloro doble: de lo cotidiano a lo extraordinario; reporte de caso y revisión de la literatura. Rev Chil Cir 2017;69(4):341-344. 